\title{
Perturbation Theory of Odd Anharmonic Oscillators
}

\author{
E. Caliceti, S. Graffi, and M. Maioli` \\ Istituto Matematico, Università di Modena, I-41100 Modena, Italy
}

\begin{abstract}
We study the perturbation theory for $H=p^{2}+x^{2}+\beta x^{2 n+1}$, $n=1,2, \ldots$ It is proved that when $\operatorname{Im} \beta \neq 0, H$ has discrete spectrum. Any eigenvalue is uniquely determined by the (divergent) Rayleigh-Schrödinger perturbation expansion, and admits an analytic continuation to $\operatorname{Im} \beta=0$ where it can be interpreted as a resonance of the problem.
\end{abstract}

\section{Introduction}

It is our purpose in the present paper to study the perturbation theory of the quantum mechanical Hamiltonian $p^{2}+x^{2}+\beta x^{3}$ or, more generally, of any odd anharmonic oscillator $p^{2}+x^{2}+\beta x^{2 n+1}, n=1,2, \ldots$. Unlike the even anharmonic oscillators, extensively studied in recent years (see e.g. Reed and Simon [16] for a review), to our knowledge the above problems did not receive so far any rigorous treatment, in spite of the fact that they are quoted in many textbooks among the simplest examples of bound state perturbation theory (see e.g. Davydov [5]). This is of course due to the well known fact that the Schrödinger operators corresponding to the above Hamiltonians admit infinitely many self-adjoint extensions when defined on $D\left(p^{2}\right) \cap D\left(x^{2 n+1}\right)$. As is known, this leads to the non-uniqueness of the quantum dynamics (for a discussion on this point, and on the connection with the behaviour of the corresponding classical motion, see e.g. Reed and Simon [15]). Although any self-adjoint extension has discrete spectrum, we shall see that the Rayleigh-Schrödinger perturbation theory near any single eigenvalue of $p^{2}+x^{2}$ exists (in the sense that the expansion is finite order by order, although divergent) but is related to the self-adjoint extensions only through a spectral concentration phenomenon.

The present situation is thus closely analogous to the Hydrogen Stark effect (with the additional complication of the non-uniqueness of the self-adjoint extensions) where the difficulty has been overcome by showing the existence of

^ Partially supported by G.N.F.M., C.N.R. 
resonances [7, 9] to which the (divergent) Rayleigh-Schrödinger perturbation expansions are uniquely associated through the Borel summability [7]. Here a somewhat analogous solution is obtained: more precisely, it will be shown that when $\operatorname{Im} \beta>0$ the formal operator $p^{2}+x^{2}+\beta x^{2 n+1}$ can be realized as a holomorphic family of compact resolvent operators in $L^{2}(\mathbb{R})$, with non-empty spectra for $|\beta|$ small enough. The Rayleigh-Schrödinger perturbation expansions are Borel summable to the eigenvalues of $p^{2}+x^{2}+\beta x^{2 n+1}$ when $|\beta|$ is small and $\pi / 8+\eta<\arg \beta<7 \pi / 8-\eta, \eta>0$. Any such eigenvalue admits an analytic continuation to $\operatorname{Im} \beta=0$, enjoying the following properties: (i) the real part is a pseudoeigenvalue of any self-adjoint extension; (ii) it satisfies the Schrödinger equation with the Gamow-Siegert boundary condition at infinity and, conversely, any Gamow-Siegert eigenvalue must be the analytic continuation to $\operatorname{Im} \beta=0$ of an eigenvalue of $p^{2}+x^{2}+\beta x^{2 n+1}, \operatorname{Im} \beta>0$; (iii) it is a second sheet pole of the scalar products of a unique generalized resolvent of the symmetric operator $p^{2}+x^{2}+\beta x^{2 n+1}$ on some dense set in $L^{2}(\mathbb{R})$.

We can thus conclude that in these problems there is a natural notion of resonance, which is uniquely associated with the bound state perturbation theory.

The exposition proceeds as follows. In Sect.II we realize the operator $p^{2}+x^{2}+\beta x^{2 n+1}$ and discuss its spectral theory, by closely following Simon's treatment [17] of $p^{2}+x^{2}+\beta x^{2 n}$. In Sect. III we treat the perturbation theory. First we prove the Borel summability and the spectral concentration of any self-adjoint extension near the unperturbed eigenvalues; then, through dilation analyticity arguments, we characterize the analytic continuation to $\operatorname{Im} \beta=0$ of all eigenvalues existing for $\operatorname{Im} \beta>0$ as the Gamow-Siegert eigenvalues of the problem as well as the second sheet poles of a unique generalized resolvent of the symmetric operator $p^{2}+x^{2}+\beta x^{2 n+1}$.

\section{The Operator $p^{2}+x^{2}+\beta x^{2 n+1}, \operatorname{Im} \beta>0$.}

As already mentioned, in this section we propose to realize the differential expression $p^{2}+x^{2}+\beta x^{2 n+1}, \operatorname{Im} \beta>0$, as an operator in $L^{2}(\mathbb{R})$, and to determine its spectral properties. To this end we shall follow as closely as possible Simon's treatment of the even case $p^{2}+x^{2}+\beta x^{2 n}[17]$.

Let us begin by stating some notations. By $L^{2}$ we will always mean the Hilbert space $L^{2}(\mathbb{R}) . p^{2}$ will always denote the self-adjoint realization of $-d^{2} / d x^{2}$ in $L^{2}$ defined in $H^{2}(\mathbb{R})$, the usual Sobolev space. By $D\left(x^{n}\right), n \in \mathbb{N}$, we shall mean the domain of the maximal multiplication operator by the function $x^{n}$ in $L^{2}$.

Definition 2.1. Let $\beta \in \mathbb{C}(\beta \in \mathbb{R}$ allowed $), \beta \neq 0$. Then $T(\beta)$ will denote the operator in $L^{2}$ defined in the following way: $D(T(\beta))=D\left(p^{2}\right) \cap D\left(x^{2 n+1}\right), T(\beta) u=\left(p^{2}+\beta x^{2 n+1}\right) u$, $u \in D(T(\beta)), n=1,2, \ldots$.

Lemma 2.2. Let $\beta \in \mathbb{R} \backslash\{0\}$. Then $T(\beta)$ is symmetric. Its closure $\bar{T}(\beta)$ has deficiency indices $(1,1)$, and admits infinitely many distinct self-adjoint extensions. All selfadjoint extensions have discrete spectrum.

Proof. We have to verify only the last assertion, since the remaining ones are well known (for a proof, see, e.g. Naimark [14]). Let $\beta>0$ and $u_{a}(x)$ be a 
solution of the differential equation $-\psi^{\prime \prime}+\beta x^{2 n+1} \psi=i \psi$ such that $\lim _{x \rightarrow-\infty}\left[u_{a}(x) \bar{u}_{a}^{\prime}(x)-u_{a}^{\prime}(x) \bar{u}_{a}(x)\right]=0$, and $v_{a}(x)$ a (linearly independent) solution which is $L^{2}$ at $+\infty$. Then it is well known (see again [14]) that the Green's function

$$
G_{a}(x, y, i)= \begin{cases}W(a)^{-1} u_{a}(x) v_{a}(y), & -\infty<x \leqq y<+\infty \\ W(a)^{-1} u_{a}(y) v_{a}(x), & -\infty<y \leqq x<+\infty\end{cases}
$$

$W(a)$ being the Wronskian of $u_{a}$ and $v_{a}$, specifies the integral kernel of $\left(T_{a}-i\right)^{-1}$, where $T_{a}$ is a self-adjoint extension of $T$. Through standard "WKB type" estimates (see again [14]) one easily finds the following asymptotic behaviours:

$$
\begin{aligned}
& \left|u_{a}(x)\right| \sim|x|^{-(2 n+1) / 4}, \quad x \rightarrow-\infty ; \\
& \left|u_{a}(x)\right| \sim|x|^{-(2 n+1) / 4} e^{\sqrt{\beta} x(2 n+3) / 2}, \quad x \rightarrow+\infty \\
& \left.v_{a}(x)|\sim| x\right|^{-(2 n+1) / 4}, \quad x \rightarrow-\infty ; \\
& \left|v_{a}(x)\right| \sim|x|^{-(2 n+1) 4} e^{-\sqrt{\beta} x^{(2 n+3) / 2}}, \quad x \rightarrow+\infty
\end{aligned}
$$

so that an easy computation yields $\int_{-\infty}^{+\infty} \int_{-\infty}^{+\infty}|G(x, y ; i)|^{2} d x d y<+\infty$. Hence $\left(T_{a}-i\right)^{-1}$ is Hilbert-Schmidt, and $T_{a}$ has discrete spectrum. Since all self-adjoint extensions have the same essential spectrum (see again [14]) the assertion is proved for $\beta>0$. If $\beta<0$, it is enough to interchange $-\infty$ with $+\infty$ in the above argument. The lemma is proved.

When $\operatorname{Im} \beta \neq 0$ the operator $T(\beta)$ has entirely different features. Considering only the $\operatorname{Im} \beta>0$ case (the $\operatorname{Im} \beta<0$ case admits of course a completely analogous treatment) we first prove a quadratic estimate which extends to all $n$ Herbst's one [9] obtained fon $n=0$.

Lemma 2.3. Let $\operatorname{Im} \beta>0$, and $u \in D(T(\beta))$. Then there are positive constants $a$ and $b$ such that:

$$
\left\|p^{2} u\right\|^{2}+|\beta|^{2}\left\|x^{2 n+1} u\right\|^{2} \leqq a\left\|\left(p^{2}+\beta x^{2 n+1}\right) u\right\|^{2}+b\|u\|^{2} .
$$

Proof. As quadratic forms on $D(T(\beta)) \otimes D(T(\beta))$ we have:

$$
\begin{aligned}
& \left(p^{2}+\bar{\beta} x^{2 n+1}\right)\left(p^{2}+\beta x^{2 n+1}\right) \\
& \quad=p^{4}+|\beta|^{2} x^{4 n+2}+\bar{\beta} x^{2 n+1} p^{2}+\beta p^{2} x^{2 n+1} \\
& \quad=p^{4}+|\beta|^{2} x^{4 n+2}+\operatorname{Re} \beta\left(x^{2 n+1} p^{2}+p^{2} x^{2 n+1}\right)+i \operatorname{Im} \beta\left[p^{2}, x^{2 n+1}\right] \\
& \quad \geqq p^{4}+|\beta|^{2} x^{4 n+2}-\left|\beta^{-1} \operatorname{Re} \beta\right|\left(p^{4}+|\beta|^{2} x^{4 n+2}\right)+(2 n+1) \operatorname{Im} \beta\left(x^{2 n} p+p x^{2 n}\right) \\
& \quad=A\left(p^{4}+|\beta|^{2} x^{4 n+2}\right)+(2 n+1) \operatorname{Im} \beta\left[\left(p+x^{2 n}\right)^{2}-p^{2}-x^{4 n}\right] \quad \text { with } \\
& \left.A=1-\left|\beta^{-1} \operatorname{Re} \beta\right|\right) \\
& \quad \geqq A\left(p^{4}+|\beta|^{2} x^{4 n+2}\right)-(2 n+1) \operatorname{Im} \beta p^{2}-(2 n+1) \operatorname{Im} \beta x^{4 n} .
\end{aligned}
$$

Now one can always find $b / 2>0$ and $\alpha, 0<\alpha<1$, such that $\alpha A p^{4}-(2 n+1) \operatorname{Im} \beta p^{2}+b / 2>0, \quad|\beta|^{2} \alpha A x^{4 n+2}-(2 n+1) \operatorname{Im} \beta x^{4 n}+b / 2>0$, whence the assertion with $a^{-1}=(1-\alpha) A$. 
As an easy consequence of this quadratic estimate we have:

Theorem 2.4. Let $\operatorname{Im} \beta>0$. Then $T(\beta)$ is closed, and its resolvent set is never empty.

Proof. The quadratic estimate (2.1) implies that $T(\beta)$ is closed on $D\left(p^{2}\right) \cap D\left(x^{2 n+1}\right)$. Now it is easily checked that the Green's function of the differential equation $-\psi^{\prime \prime}+\beta x^{2 n+1} \psi=0$ with $L^{2}$ boundary conditions at $\pm \infty$ is given by:

$$
G(x, y)= \begin{cases}\pi^{-1} \sqrt{x y} H_{1 /(2 n+3)}^{(2)}\left(x^{\prime}\right) H_{1 /(2 n+3)}^{(1)}\left(y^{\prime}\right), & -\infty<x \leqq y<+\infty \\ \pi^{-1} \sqrt{x y} H_{1 /(2 n+3)}^{(2)}\left(y^{\prime}\right) H_{1 /(2 n+3)}^{(1)}\left(x^{\prime}\right), & -\infty<y \leqq x<+\infty\end{cases}
$$

where $x^{\prime}=2 /(2 n+3) i \sqrt{\beta x^{(2 n+3) / 2}}, y^{\prime}=2 /(2 n+3) i \sqrt{\beta y^{(2 n+3) / 2}}$, and $H^{(2),(1)}(\cdot)$ are the Hankel functions. Taking into account the known asymptotic behaviour of such functions (see e.g. [6]) a simple computation shows that $\int_{-\infty}^{+\infty} \int_{-\infty}^{+\infty}|G(x, y)|^{2} d x d y<+\infty$. If $K$ denotes the Hilbert-Schmidt operator in $L^{2}$ generated by $G$, one immediately has $T(\beta) K=I$, and this proves the result.

Corollary 2.5. Let $\operatorname{Im} \beta>0$. Then $T(\beta)$ is a holomorphic family of type $A$ of operators in $L^{2}$.

Proof. $D(T(\beta))$ does not depend on $\beta$, and the resolvent of $T(\beta)$ is Hilbert-Schmidt by the former result. Since $T(\beta) u$ is of course a holomorphic vector valued function of $u$ for any $u \in D(T(\beta)), T(\beta)$ is a holomorphic family of type $A$ by definition (see [16], XII. 2). This proves the corollary.

Consider now in $L^{2}$ the operator $H_{0}(\gamma),|\arg \gamma|<\pi$, defined by:

$$
D\left(H_{0}(\gamma)\right)=D\left(p^{2}\right) \cap D\left(x^{2}\right), \quad H_{0}(\gamma) u=\left(p^{2}+\gamma x^{2}\right) u, \quad u \in D\left(H_{0}(\gamma)\right) .
$$

The following result is proved in Simon [17].

Lemma 2.6. $H_{0}(\gamma)$ is a holomorphic family of type $A$ of compact resolvent operators. The spectrum of $H_{0}(\gamma)$ is given by the sequence of the simple eigenvalues $E_{i}^{0}(\gamma)=\sqrt{\gamma}(2 i+1), i=0,1, \ldots$

Definition 2.7. Let $\beta \in \mathbb{C}(\beta \in \mathbb{R}$ allowed). By $H(\beta)$ we shall mean the differential operator in $L^{2}(\mathbb{R})$ defined as follows :

$$
\begin{aligned}
D(H(\beta))= & D\left(p^{2}\right) \cap D\left(x^{2 n+1}\right), H(\beta) u=\left(p^{2}+x^{2}\right) u+\beta x^{2 n+1} u \\
& \cdot u \in D(H(\beta))=D\left(H_{0}(\gamma)\right) \cap D\left(x^{2 n+1}\right) .
\end{aligned}
$$

When $\beta \in \mathbb{R} \backslash\{0\}, H(\beta)$ has properties completely analogous to those of $T(\beta)$ listed in Lemma 2.2, since its proof applies also to $H(\beta)$ without modifications. To define $H(\beta)$ for $\operatorname{Im} \beta \neq 0$ we need a second quadratic estimate (compare with [17], Lemma II. 9.1).

Lemma 2.8. Let $u \in D\left(p^{2}\right) \cap D\left(x^{2 n+1}\right)$, and $\operatorname{Im} \beta>0$. Then there are positive constants $a$ and $b$ such that

$$
\left\|\left(p^{2}+x^{2}\right) u\right\|^{2}+|\beta|^{2}\left\|x^{2 n+1} u\right\|^{2} \leqq a\|H(\beta) u\|^{2}+b\|u\|^{2} .
$$


Proof. Again as quadratic forms on $D(H(\beta)) \otimes D(H(\beta))$ we can write:

$$
\begin{aligned}
\left(p^{2}+\right. & \left.x^{2}+\bar{\beta} x^{2 n+1}\right)\left(p^{2}+x^{2}+\beta x^{2 n+1}\right) \\
= & p^{4}+x^{4}+|\beta|^{2} x^{4 n+2}+2 \operatorname{Re} \beta x^{2 n+3} \\
& +p^{2} x^{2}+x^{2} p^{2}+\operatorname{Re} \beta\left(p^{2} x^{2 n+1}+x^{2 n+1} p^{2}\right)+i \operatorname{Im} \beta\left[p^{2}, x^{2 n+1}\right] \\
\geqq & p^{4}+x^{4}+|\beta|^{2} x^{4 n+2}+2 \operatorname{Re} \beta x^{2 n+3}-\left|\beta^{-1} \operatorname{Re} \beta\right|\left(p^{4}+|\beta|^{2} x^{4 n+2}\right) \\
& +\left[p,\left[p, x^{2}\right]\right]+2 p x^{2} p+(2 n+1) \operatorname{Im} \beta\left(x^{2 n} p+p x^{2 n}\right) \\
\geqq & \left(\operatorname{with} A=1-\left|\beta^{-1} \operatorname{Re} \beta\right|\right) \geqq A\left(p^{4}+|\beta|^{2} x^{4 n+2}\right)+x^{4}+2 \operatorname{Re} \beta x^{2 n+3} \\
& -2+(2 n+1) \operatorname{Im} \beta\left[\left(p+x^{2 n}\right)^{2}-p^{2}-x^{4 n}\right] \\
\geqq & A\left(p^{4}+|\beta|^{2} x^{4 n+2}\right)+x^{4}+2 \operatorname{Re} \beta x^{2 n+3}-2-(2 n+1) \operatorname{Im} \beta p^{2}-(2 n+1) \operatorname{Im} \beta x^{4 n}
\end{aligned}
$$

whence the assertion by proceeding exactly as in Lemma 2.3 .

Hence, in complete analogy with Theorem 2.4 and Corollary 2.5, we have:

Theorem 2.9. Let $\operatorname{Im} \beta>0$. Then $H(\beta)$ is a holomorphic family of type $A$ of operators in $L^{2}$.

In order to determine the nature of the spectrum of $H(\beta)$ and its stability as $\beta \rightarrow 0$ we need the analog of a further result of Simon, namely:

Theorem 2.10. Let $\gamma$ belong to the half-plane $\operatorname{Re} \gamma>0$. Then $H(\gamma,|\beta|)$ $=H_{0}(\gamma)+i|\beta| x^{2 n+1}$ defined on $D\left(p^{2}\right) \cap D\left(x^{2 n+1}\right)$ is a holomorphic family of type $A$ (in $\gamma$ ). For any compact $\Omega$ of the half-plane there are $a>0$ and $b>0$ such that

$$
\left\|x^{2} u\right\|^{2} \leqq a\left\|\left(p^{2}+\gamma x^{2}+i|\beta| x^{2 n+1}\right) u\right\|^{2}+b\|u\|^{2}
$$

$u \in D\left(p^{2}\right) \cap D\left(x^{2 n+1}\right), \gamma \in \Omega, 0<|\beta| \leqq 1, a$ and $b$ being independent of $\gamma$ in $\Omega$.

Proof. Let us prove the assertion through a further quadratic estimate, i.e:

$$
\begin{aligned}
& \left\|\left(p^{2}+i|\beta| x^{2 n+1}\right) u\right\|^{2}+|\gamma|^{2}\left\|x^{2} u\right\|^{2} \\
& \quad \leqq a\left\|\left(p^{2}+\gamma x^{2}+i|\beta| x^{2 n+1}\right) u\right\|^{2}+b\|u\|^{2}, u \in D\left(p^{2}\right) \cap D\left(x^{2 n+1}\right),
\end{aligned}
$$

where the positive constants $a$ and $b$ can be chosen independent of $\gamma$ in $\Omega$. Remark that (2.6) implies (2.5) and, by the quadratic estimate of Lemma 2.3, it also implies

$$
|\beta|^{2}\left\|x^{2 n+1} u\right\|^{2} \leqq a^{\prime}\left\|\left(p^{2}+\gamma x^{2}+i|\beta| x^{2 n+1}\right) u\right\|^{2}+b^{\prime}\|u\|^{2}
$$

again with $a^{\prime}$ and $b^{\prime}$ independent of $\gamma$ in $\Omega, u \in D\left(p^{2}\right) \cap D\left(x^{2 n+1}\right)$. To see (2.6), always as quadratic forms on $D\left(p^{2}\right) \cap D\left(x^{2 n+1}\right) \otimes D\left(p^{2}\right) \cap D\left(x^{2 n+1}\right)$ let us write:

$$
\begin{aligned}
& \left(p^{2}+\bar{\gamma} x^{2}-i|\beta| x^{2 n+1}\right)\left(p^{2}+\gamma x^{2}+i|\beta| x^{2 n+1}\right)=\left(p^{2}-i|\beta| x^{2 n+1}\right)\left(p^{2}+i|\beta| x^{2 n+1}\right) \\
& \quad+|\gamma|^{2} x^{4}+\operatorname{Re} \gamma\left(p^{2} x^{2}+x^{2} p^{2}\right)+i \operatorname{Im} \gamma\left(p^{2}-i|\beta| x^{2 n+1}\right) x^{2}-i \operatorname{Im} \gamma x^{2}\left(p^{2}+i|\beta| x^{2 n+1}\right) \\
& =\left|\gamma^{-1} \operatorname{Im} \gamma\right|\left(p^{2}-i|\beta| x^{2 n+1} \mp i|\gamma| x^{2}\right)\left(p^{2}+i|\beta| x^{2 n+1} \pm i|\gamma| x^{2}\right) \\
& \quad+\left(1-\left|\gamma^{-1} \operatorname{Im} \gamma\right|\right)\left[\left(p^{2}-i|\beta| x^{2 n+1}\right)\left(p^{2}+i|\beta| x^{2 n+1}\right)+|\gamma|^{2} x^{4}\right]-2 \operatorname{Re} \gamma+2 \operatorname{Re} \gamma p x^{2} p \\
& \geqq \\
& \left(\text { for some } A<1-\left|\gamma^{-1} \operatorname{Im} \gamma\right|\right) A\left(p^{2}-i|\beta| x^{2 n+1}\right)\left(p^{2}+i|\beta| x^{2 n+1}\right)+A|\gamma|^{2} x^{4}-B
\end{aligned}
$$

whence the assertion, with the required uniformities, with $a=A^{-1}, b=B A^{-1}$, for some $B>2 \operatorname{Re} \gamma$. 
Of course in this case as well a basic tool is the Symanzik scaling property. Exactly as in [17], Theorem II.2.1, one has

Theorem 2.11. Let $\operatorname{Re} \gamma>0, \operatorname{Im} \beta>0, \lambda>0$. Then if $E(\gamma, \beta)$ is an eigenvalue of $H(\gamma, \beta)$, we have:

$$
E(\gamma, \beta)=\lambda E\left(\gamma \lambda^{-2}, \beta \lambda^{-(2 n+3) / 2}\right) .
$$

Remark. (2.8) extends to $\lambda$ complex by analytic continuation, whenever such continuation exists.

We are now in position to prove the key result of this section, namely the norm resolvent convergence of $H(\beta)$ to $H_{0}$ as $|\beta| \rightarrow 0, \operatorname{Im} \beta>0$. Let us proceed as in Lemma II.9.3 of [17]. First remark that, by a scaling of the phase of $\beta,[H(\beta)$ is a holomorphic family in $\beta$ ] we can study $H(\beta)$ by looking instead at $H(\gamma,|\beta|)$, where $|\gamma|=1, \operatorname{Re} \gamma>0, \gamma=\exp [i(2 \pi /(2 n+3)-4 \arg \beta /(2 n+3))]$. The condition $\operatorname{Re} \gamma>0$ implies of course $-(2 n-1) \pi / 8<\arg \beta<(2 n+7) \pi / 8$. As a second step, let us state a simple remark under the form of a lemma.

Lemma 2.12. $H(\gamma,|\beta|)$ is uniformly sectorial when $\gamma$ ranges in any compact $\Omega$ contained in the right half-plane, i.e. the union $U$ of the numerical ranges of $H(\gamma,|\beta|), \gamma \in \Omega$, is contained in a half-plane.

Proof. We have:

$$
\operatorname{Re}\langle H(\gamma,|\beta|) u, u\rangle=\operatorname{Re}\left\langle\left(p^{2}+\gamma x^{2}\right) u, u\right\rangle>0 \quad \text { when } \quad \operatorname{Re} \gamma>0, u \in D(H(\gamma,|\beta|)) .
$$

Theorem 2.13. Let $\gamma$ belong to the half-plane $\operatorname{Re} \gamma>0$, and let $R_{\gamma}(|\beta|, E)$ $=[H(\gamma,|\beta|)-E]^{-1}, R_{\gamma}(0, E)=\left[H_{0}(\gamma)-E\right]^{-1}$. Then $\left\|R_{\gamma}(|\beta|, E)-R_{\gamma}(0, E)\right\| \rightarrow 0$ as $|\beta| \rightarrow 0$, uniformly on compacts with respect to $\gamma$.

Proof. Let us consider here only the $n=1$ case, since it is a straightforward adaptment of the argument of [17], Lemma II.10.1. The general case requires a more complicated argument, to be described in Appendix. Since it is enough to prove the convergence for a single value of $E$, choose an $E$ at a positive distance $c$ from the union $U$ of the numerical ranges of $H(\gamma,\{\beta \mid)$, which by Lemma 2.12 is possible independently of $\gamma$ and $|\beta| \geqq 0$. Then we have $\left\|R_{\gamma}(|\beta|, E)\right\|<c^{-1}$, uniformly with respect to $\gamma$ and $|\beta| \geqq 0$. Next, by the second resolvent formula $R_{\gamma}(|\beta|, E)$ $-R_{\gamma}(0, E)=-i|\beta|\left[R_{\gamma}(0, E) x\right]\left[x^{2} R_{\gamma}(|\beta|, E)\right]$. By Lemma II.9.4 of [17], we have $\mid R_{\gamma}(0, E) x \|<a+c(b+a|E|)$ for some $a>0, b>0$ independent of $\gamma$. By Theorem 2.10 also $x^{2} R_{\gamma}(|\beta|, E)$ is bounded for $|\beta| \geqq 0$, uniformly on compacts in $\gamma$. Hence there is $c^{\prime}>0$ such that $\left\|R_{\gamma}(|\beta|, E)-R_{\gamma}(0, E)\right\| \leqq c^{\prime}|\beta| \rightarrow 0$ as $|\beta| \rightarrow 0$ and the Theorem is proved for $n=1$. The proof for all $n$ is given in Appendix.

Corollary 2.14. Let $\operatorname{Re} \gamma>0$. Then $H(\gamma,|\beta|)$ has compact resolvent for any $\gamma$ and any $\beta$, and the same is true for $H(\beta), \operatorname{Im} \beta>0$.

Proof. Since $R_{\gamma}(0, E)$ is compact, there are values of $|\beta|$ so small that $R_{\gamma}(|\beta|, E)$ is compact. Since $H(\gamma,|\beta|)$ and $H(\beta)$ are holomorphic families of type $A$, the assertion is a consequence of a well known result (see [11], Theorem VII.2.4).

Thus the spectra of $H(\gamma,|\beta|)$ and $H(\beta), \operatorname{Im} \beta>0$, are discrete. The norm resolvent convergence ensures that, at least for $|\beta|$ small, they are not empty. The following result has the same proof of Theorem II.10.2 of [17]. 
Theorem 2.15. Let $i \in \mathbb{N}_{0}$, and let $\Gamma$ ba a compact subset of the half-plane $\operatorname{Re} \gamma>0$. Then there is a $B>0$ such that for $|\beta|<B$ and $\gamma \in \Gamma, H(\gamma,|\beta|)$ has exactly one eigenvalue $E_{i}(\gamma,|\beta|)$ near $(2 i+1) \gamma^{1 / 2}$. As $|\beta| \rightarrow 0$ one has $E_{i}(\gamma,|\beta|) \rightarrow(2 i+1) \gamma^{1 / 2}$, uniformly for $\gamma \in \Gamma$.

Then, again as in [17] (Theorem II. 10.3) by rescaling the phase of $\beta$ one has:

Corollary 2.16. Let $i \in N_{0}$ and $\varepsilon>0$ be given. Then there is a $B>0$ such that for $|\beta|<B, \operatorname{Im} \beta>0 H(\beta)$ has exactly one eigenvalue near $2 i+1$. Such eigenvalues are analytic functions of $\beta$ for $|\beta|<B, \operatorname{Im} \beta>0$, and admit a (many valued) analytic continuation across the real axis to the whole sector $|\beta|<B,-(2 n-1) \frac{\pi}{8}$ $+\varepsilon<\arg \beta<(2 n+7) \frac{\pi}{8}-\varepsilon$.

Remark. It will be proved in the next section that the analytic continuation of the functions $E_{i}(\beta)$ across the real axis is not single valued. However we will not attempt here to discuss the global analytic structure of $E_{i}(\beta)$ on an algebraic Riemann surface, in analogy with the results discovered by Bender and $\mathrm{Wu}$ [4] for the even anharmonic oscillators, later rigorously justified by Simon [17], Loeffel et al. [12], Loeffel and Martin [13].

\section{Resonances and Perturbation Theory}

Let us now begin by examining the perturbation theory of the problem. From now on, let us denote $H_{0}=p^{2}+x^{2}, V=x^{2 n+1}$, so that $H(\beta)=H_{0}+\beta V$, defined as above. We first state a lemma on the perturbation series itself whose content is essentially well known.

Lemma 3.1. Let $E(\beta)$ denote an arbitrary eigenvalue of $H(\beta), \operatorname{Im} \beta>0,|\beta|<B$. Then the function $E(\beta)$ has a formal (i.e. divergent) Taylor expansion in powers of $\beta^{2}$ near $\beta=0$ :

$$
E(\beta) \sim \sum_{0}^{\infty} a_{2 k} \beta^{2 k}, \quad a_{0}=2 i+1,
$$

where the coefficients $a_{2 k}, k=1,2, \ldots$, are given by the Rayleigh-Schrödinger perturbation theory.

Proof. Remark that $D(V) \supset C^{\infty}\left(H_{0}\right)=\bigcap_{n=1}^{\infty} D\left(H_{0}^{n}\right)$, and that $V$ leaves $C^{\infty}\left(H_{0}\right)$ invariant. In addition $C^{\infty}\left(H_{0}\right) \subset D(H(\beta))$, and of course $H(\beta) \psi=H_{0} \psi+\beta V \psi$ if $\psi \in C^{\infty}\left(H_{0}\right)$. Hence, by the norm resolvent convergence of Theorem 2.13 we can conclude that all assumptions of Theorem XII.14 of [16] are satisfied, so that $E(\beta)$ has the Taylor expansion (3.5), which is in addition uniformly asymptotic to $E(\beta)$ in any sector $-(2 n-1) \pi / 8+\varepsilon<\arg \beta<(2 n+7) \pi / 8-\varepsilon, \varepsilon>0$.

The fact that only the even terms are nonzero is an easy consequence of the invariance of the problem under the transformations $x \rightarrow-x, \beta \rightarrow-\beta$. Finally, the divergence of the series follows by a diagram counting argument quite standard in this framework, so that it will be omitted here. 
The one-to-one relationship between the eigenvalue $E(\beta)$ and the (divergent) Rayleigh-Schrödinger perturbation expansion is guaranteed by the Borel summability, which we proceed now to prove.

Theorem 3.2. Let $E(\beta)$ be an eigenvalue of $H(\beta)=H_{0}+\beta V, \operatorname{Im} \beta>0$. Then there are $C>0$ and $\eta>0$ such that the corresponding Rayleigh-Schrödinger perturbation expansion is Borel summable to the function $E(\beta)$ in the region $|\beta|<C$, $\pi / 8+\eta<\arg \beta<7 \pi / 8-\eta$.

Proof. Again, let us adapt a quite standard argument (see e.g. [16], XII.4). First remark that, by Corollary 2.16 , the function $E\left(\beta^{2}\right)$ is analytic in the Riemann surface sector $0<|\beta|^{2}<B^{2}, \quad-(2 n-1) \pi / 4+\varepsilon<\arg \left(\beta^{2}\right)<(2 n+7) \pi / 4-\varepsilon$ so that $E\left(e^{-i \pi} \beta^{2}\right)$ is analytic in the sector $0<|\beta|^{2}<B^{2},\left|\arg \left(\beta^{2}\right)\right|<(2 n+3) \pi / 4-\varepsilon$. Then the norm resolvent convergence of Theorem 2.13 allows to apply the very same argument of [16], XII.4, Example 1, to deduce the bound ( $D$ and $\sigma$ being positive constants)

$$
\left\|\left[V\left(H_{0}-E\right)^{-1}\right]^{N+1} \Omega\right\| \leqq D \sigma^{N+1} \Gamma\left(\frac{n}{2} N+1\right), \quad N=1,2, \ldots
$$

for $\left|E-E_{0}\right|=1 / 2$ ( $E_{0}$ the unperturbed eigenvalue, $\Omega$ the corresponding eigenvector) which yields (see again [16], XII.4) the strong asymptotic bound

$$
\left|E\left(e^{-i \pi} \beta^{2}\right)-\sum_{0}^{N}(-1)^{k} a_{k} \beta^{2 k}\right| \leqq D \sigma^{N+1}(n N+1)\left|\beta^{2}\right|^{n} .
$$

This last bound, together with the analyticity of $E\left(e^{-i \pi} \beta^{2}\right)$ in the the above sector, yields by Watson's theorem (see again [16], XII.4) the Borel summability of $\sum_{0}^{\infty}(-1)^{k} \mathrm{a}_{k} \beta^{2 k}$ to $E\left(e^{-i \pi} \beta^{2}\right)$ for $|\beta|<C,\left|\arg \left(e^{-i \pi} \beta^{2}\right)\right|<3 \pi / 4-\eta, \eta>0$, and this in turn implies the Borel summability of the original perturbation expansion to $E(\beta)$ for $|\beta|<C, \pi / 8+\eta<\arg \beta<7 \pi / 8-\eta$.

This proves the theorem.

Remarks. (a) When $n>1$, by Borel summability we mean for short the generalized Borel summability of order $n$ of Leroy (see e.g. [8]).

(b) All eigenvalues existing for $|\beta|<B, \arg \beta=\pi / 2$, are real. This is due to the Borel summability since all terms of the series are real when $\beta$ is purely imaginary.

We can now prove the result on the multivaluedness of the analytic continuation of any function $E(\beta)$ across the real axis mentioned in the remark after Corollary 2.16 .

Theorem 3.3. Let $|\beta|<B, \operatorname{Im} \beta>0$, and $E(\beta)$ be an eigenvalue of $H(\beta)$. Then the analytic continuation of $E(\beta)$ across the real axis is not single valued, and $\lim _{\operatorname{Im} \beta \rightarrow 0}$ $\operatorname{Im} E(\beta) \neq 0$ except for a discrete set of points on the real line.

Proof. We have seen that $E\left(\beta^{2}\right)$ is not analytic at $\beta=0$, since its Taylor expansion is divergent. However $E\left(\beta^{2}\right)$ is analytic in the punctured sector $-(2 n-1) \pi / 4+\varepsilon$ $<\arg \beta^{2}<(2 n+7) \pi / 4-\varepsilon, \varepsilon>0$, with finite limits as $|\beta| \rightarrow 0$ within the sector. Hence 
the singularity at zero of $E\left(\beta^{2}\right)$ must be a branch point, so that $E(\beta)$ will have a branch cut along the real axis. Now it is easy to see that $\bar{E}(\beta)=E(\bar{\beta})$, so that $2 i \lim _{\operatorname{Im} \beta \rightarrow 0_{+}} \operatorname{Im} E(\beta)$ yields the discontinuity across the cut. Hence $\operatorname{Im} E(\beta)$, which is analytic along the real axis, can vanish only on a discrete set of points, since otherwise $E(\beta)$ would be single valued. This proves the theorem.

Remark. Let $\beta \in \mathbb{R} \backslash\{0\}$. Then the series (3.1) is real since $a_{k}$ is real for any $k$. Hence $\operatorname{Im} E(\beta)$ has zero asymptotic expansion as $\beta \rightarrow 0$, i.e. $\operatorname{Im} E(\beta)=o\left(\beta^{n}\right)$ for all $n$ as $\beta \rightarrow 0$, and $\operatorname{Re} E(\beta)$ has an asymptotic expansion to all orders in $\beta$ given by the perturbation series (3.1).

Let us now verify that, given any eigenvalue $E(\beta), \operatorname{Im} \beta>0$, the real part of its analytical continuation to $\operatorname{Im} \beta=0$ is a pseudoeigenvalue of any self-adjoint extension of $\bar{H}(\beta)$.

Lemma 3.4. Let $\beta \in \mathbb{R} \backslash\{0\}$, and $H_{a}(\beta)$ be an arbitrary self-adjoint extension of $\bar{H}(\beta)$. Then $H_{a}(\beta)$ converges strongly in the generalized sense to $H_{0}=p^{2}+x^{2}, D\left(H_{0}\right)$ $=D\left(p^{2}\right) \cap D\left(x^{2}\right)$, as $\beta \rightarrow 0$.

Proof. Let $u \in C_{0}^{\infty}(\mathbb{R})$. Then $H_{a}(\beta) u \rightarrow H_{0} u$ as $\beta \rightarrow 0$. Since $C_{0}^{\infty}(\mathbb{R})$ is a core of $H_{0}$, and $H_{0}$ and $H_{a}$ are both self-adjoint, the assertion is true by Corollary VIII.1.6 of [11].

As a consequence we have:

Theorem 3.5. Let $\beta \in \mathbb{R} \backslash\{0\}$. Then as $\beta \rightarrow 0$ the spectrum of any self-adjoint extension $H_{a}(\beta)$ of $\bar{H}(\beta)$ is asymptotically concentrated to all orders in $\beta$ near the eigenvalues of $H_{0}$. If $E(\beta)$ is an eigenvalue of $H(\beta), \operatorname{Im} \beta>0$, the real part of its analytic continuation to $\operatorname{Im} \beta=0$ is a pseudoeigenvalue (to all orders in $\beta$ ) of any self-adjoint extension $H_{a}(\beta)$, and the imaginary part yields the width of the spectral concentration.

Proof. By Lemma 3.4 and the well known spectral decomposition of $H_{0}$, the conditions of Theorem VIII.5.4 and Remark VIII.5.6 of [11] are trivially verified in the present case. Hence (see also [16], XII.5) if $E_{0}$ is an arbitrary eigenvalue of $H_{0}$ and $I \subset \mathbb{R}$ any open interval such that $\sigma\left(H_{0}\right) \cap \bar{I}=\left\{E_{0}\right\}$, for all $n=0,1,2, \ldots$ there is a function $f(\beta)$, with the property $f^{(n)}(\beta)|\beta|^{-n} \rightarrow 0$ as $\beta \rightarrow 0$, such that the part of the spectrum of $H_{a}(\beta)$ in $I$ is asymptotically concentrated in

$$
\left(\sum_{k=0}^{n} a_{2 k} \beta^{2 k}-f^{(n)}(\beta), \sum_{k=0}^{n} a_{2 k} \beta^{2 k}+f^{(n)}(\beta)\right),
$$

where $\sum a_{2 k} \beta^{2 k}$ is the Rayleigh-Schrödinger expansion (3.1). Since for $\operatorname{Im} \beta=0$ $\operatorname{Re} E(\beta)$ admits this series as an asymptotic expansion to all orders in $\beta$ as $\beta \rightarrow 0$, it is by definition a pseudoeigenvalue (to all orders) of any self-adjoint extension $H_{a}$. In addition $\operatorname{Im} E(\beta)$ has zero asymptotic expansion as $\beta \rightarrow 0, \beta \in \mathbb{R}$. Hence we can take $\operatorname{Im} E(\beta)=f^{(n)}(\beta)$ for all $n$ and the theorem is proved.

Let us now proceed to prove a second characterization of the analytic continuation to $\operatorname{Im} \beta=0$ of any eigenvalue of $H(\beta), \operatorname{Im} \beta>0$. If, instead of imposing a boundary condition at infinity in order to get a self-adjoint operator out of the differential expression $p^{2}+x^{2}+\beta x^{2 n+1}, \beta \in \mathbb{R} \backslash\{0\}$, one imposes the GamowSiegert "resonance" boundary condition (see e.g. [18]), the resulting complex 
eigenvalues are nothing else than the above analytically continued eigenvalues. To this end, and also for later purposes, use will be made of the dilation analyticity technique, so that let us first state some notation.

Consider in $L^{2}(\mathbb{R})$ the formal differential operator

$$
\begin{aligned}
H(\beta, \theta) & =-e^{-\theta} d^{2} / d x^{2}+e^{\theta} x^{2}+\beta e^{\left(\frac{2 n+1}{2}\right) \theta} x^{2 n+1} \\
& =e^{-\theta}\left(-d^{2} / d x^{2}+e^{2 \theta} x^{2}+\beta e^{\left(\frac{2 n+3}{2}\right) \theta} x^{2 n+1}\right) .
\end{aligned}
$$

(Notice that this amounts to set the scaling parameter $\lambda$ equal to $e^{-\theta}$.) By the results of Sect. 2 we know that $H(\beta, \theta)$, when defined on $D\left(p^{2}\right) \cap D\left(x^{2 n+1}\right)$, is a holomorphic family of type A with compact resolvents for $\operatorname{Re}\left(e^{2 \theta}\right)>0$, $\operatorname{Im}\left(\beta e^{\left(\frac{2 n+3}{2}\right) \theta}\right)>0$. In addition the eigenvalues of $H(\beta, \theta)$ (which exist for $|\beta|$ small enough) do not depend on $\theta$ by the Symanzik scaling property, and when $\operatorname{Im} \beta>0$ coincide with the eigenvalues of $H(\beta), \operatorname{Im} \beta>0$, because for $\theta$ real the scaling by $e^{-\theta}$ is a unitary operation. Hence from now on we shall always take $\beta \in \mathbb{R}_{+}$, so that $H(\beta, \theta)$ will be a holomorphic family in $\theta$ for all $\theta$ in the open strip $0<\operatorname{Im} \theta$ $<\min \left(\frac{\pi}{4}, \frac{2 \pi}{2 n+3}\right)$. (If $\beta \in \mathbb{R}_{-}$the modification of the argument is obvious.) For $|\beta|<B H(\beta, \theta)$ has eigenvalues, which are given by the analytic continuation to $\operatorname{Im} \beta=0$ of the eigenvalues of $H(\beta), \operatorname{Im} \beta>0$.

Let us now state some additional notations. $H_{a}(\beta)$ will denote an arbitrary selfadjoint extension of $\bar{H}(\beta)$, the closure of the symmetric operator $p^{2}+x^{2}+\beta x^{2 n+1}$ defined on $D\left(p^{2}\right) \cap D\left(x^{2 n+1}\right)$. Let $\theta$ be complex, $\theta=\theta_{1}+i \theta_{2}$. By $H_{a}\left(\beta, \theta_{1}\right)$ we shall mean the unitary image of $H_{a}(\beta)$ under the real dilation transformation $\left(U\left(\theta_{1}\right) f\right)(x)=e^{(1 / 2) \theta_{1}} f\left(e^{\theta_{1}} x\right), f \in L^{2}$, i.e.:

$$
H_{a}\left(\beta, \theta_{1}\right)=U\left(\theta_{1}\right) H_{a}(\beta) U\left(\theta_{1}\right)^{-1} \text {. }
$$

Let furthermore $H\left(\beta, \theta_{1}\right)=U\left(\theta_{1}\right) H(\beta) U\left(\theta_{1}\right)^{-1}$ denote the unitary image of $H(\beta)$ under $U\left(\theta_{1}\right)$, and let $\bar{H}\left(\beta, \theta_{1}\right)=U\left(\theta_{1}\right) \bar{H}(\beta) U\left(\theta_{1}\right)^{-1}$ be its closure. Of course on $D\left(p^{2}\right) \cap D\left(x^{2 n+1}\right)$ one has:

$$
H\left(\beta, \theta_{1}\right)=e^{-\theta_{1}}\left(-d^{2} / d x^{2}+e^{2 \theta_{1}} x^{2}+\beta e^{(2 n+3) \theta_{1} / 2} x^{2 n+1}\right) .
$$

Let us finally specify what we mean by the Gamow-Siegert boundary condition in this context. Since all solutions of the differential equations $\left(p^{2}+x^{2}+\beta x^{2 n+1}\right) \psi$ $=E \psi$ are of course entire functions of $x$, considered as a complex variable, the following definition is allowed.

Definition 3.6. Let $\beta \in \mathbb{R}_{+}$. We say that $E(\beta)$ is a Gamow-Siegert eigenvalue of $H(\beta)$ if there is a $\psi(x, \beta)$ such that:

(i) $\left(p^{2}+x^{2}+\beta x^{2 n+1}\right) \psi=E(\beta) \psi, x \in \mathbb{R}$;

(ii) for any $\varepsilon>0$ there is $\tau(\varepsilon)$ such that $e^{\tau|x|}|\psi(x)|$ is bounded as $|x| \rightarrow \infty$ in any direction within the sector $0 \leqq \arg (x)<\pi-\varepsilon$. The results of Sect. II allow a characterization of all such Gamow-Siegert eigenvalues. More precisely, we have:

Theorem 3.7. Let $\beta \in \mathbb{R}_{+}$. Then $E(\beta)$ is a Gamow-Siegert eigenvalue of $H(\beta)$ in the above sense if and only if it is an eigenvalue of $H(\beta, \theta), 0<\operatorname{Im} \theta<\min \left(\frac{\pi}{4}, \frac{2 \pi}{2 n+3}\right)$. 
Proof. Let $x \in R$. The Symanzik scaling immediately implies that $\psi(x)$ solves $\left(p^{2}+x^{2}+\beta x^{2 n+1}\right) \psi=E(\beta) \psi$ if and only if $\psi\left(\left(e^{\theta / 2} x\right)\right.$ solves

$$
e^{-\theta}\left(p^{2}+e^{2 \theta} x^{2}+\beta e^{\frac{2 n+3}{2}} x^{2 n+1}\right) \psi\left(e^{\theta / 2} x\right)=E(\beta) \psi\left(e^{\theta / 2} x\right) .
$$

If we take $\beta$ complex with arbitrarily small positive imaginary part the assertion is a consequence of the known spectral properties of $H(\beta, \theta)$, since by a standard WKB type estimate any eigenvector of $H(\beta, \theta)$ is $O|x|^{-\frac{2 n+1}{4}} e^{-|x| \frac{2 n+3}{4}}$ as $|x| \rightarrow \infty$ in any direction contained in $0 \leqq \arg (x)$ $<\pi-(1 / 2) \operatorname{Im} \theta$.

Let us finally come to a third characterization of the eigenvalues of $H(\beta, \theta)$, $\beta \in \mathbb{R}_{+}$. We will see that there is a unique generalized resolvent $\mathscr{R}(E)$ of the symmetric operator $H(\beta)$ and a dense set $S$ of dilation analytic vectors such that if $\psi \in S$ any function $\langle\psi, \mathscr{R}(\mathrm{E}) \psi\rangle$ has a meromorphic continuation from the upper half-plane $\operatorname{Im} E>0$ through the cut along the real axis. The poles of the meromorphic continuation coincide with the eigenvalues of $H(\beta, \theta)$.

To this purpose, let us briefly recall the notions of generalized resolvent and of spectral function of a symmetric operator (for a detailed account see e.g. $[1,2]$; the reader is referred to [1] also for the connection between these notions and the classical moment problem, to be exploited later on).

An operator-valued function $F(t): \mathbb{R} \rightarrow B(X)[X$ a complex Hilbert space, $B(X)$ the space of all bounded operators on $X]$ is a spectral function of a closed symmetric operator $A: X \rightarrow X$ of deficiency indicies $(m, n)(m<\infty, n<\infty)$ if:

(a) $F(-\infty)=0 ; F(+\infty)=I$. (b) $F(t+0)=F(t), t \in \mathbb{R}$.

(c) If $s>t, F(s)-F(t) \geqq 0$. (d) If $u \in D(A), v \in X$,

$$
\langle A u, v\rangle=\int_{-\infty}^{+\infty} t d\langle F(t) u, v\rangle, \quad\|A u\|^{2}=\int_{-\infty}^{+\infty} t^{2} d\langle F(t) u, u\rangle .
$$

An operator-valued function $\mathscr{R}(E): \mathbb{C} \backslash \mathbb{R} \rightarrow B(X)$ is a generalized resolvent of a closed symmetric operator $A: X \rightarrow X$ if and only if it can be represented in the form

$$
\mathscr{R}(E)=\int_{-\infty}^{+\infty}(t-E)^{-1} d F(t)
$$

where $F(t)$ is a spectral function of $A$, and the integral is intended in the weak sense. If $F(t)$ is an orthogonal spectral family then $\mathscr{R}(E)$ is the resolvent of a self-adjoint extension of $A$.

The exact statement of the above mentioned result is expressed by the following theorem and its corollaries.

Theorem 3.8. There is a dense set of dilation analytic vectors (for $\left.|\operatorname{Im} \theta|<\min \left(\frac{\pi}{4}, \frac{2 \pi}{2 n+3}\right)\right)$ and a unique spectral function $F(t)$ of the closed symmetric operator $\bar{H}(\beta)$ such that if $\psi \in S$ any function

$$
f_{\psi}(E)=\langle\psi, \mathscr{R}(E) \psi\rangle \equiv \int_{-\infty}^{+\infty}(t-E)^{-1} d\langle\psi, F(t) \psi\rangle
$$


which is a priori defined as an analytic function of $E$ in the upper half-plane $\operatorname{Im} E>0$, has a meromorphic continuation to the lower half-plane $\operatorname{Im} E \leqq 0$. The set of singularities $\left\{E \mid f_{\psi}\right.$ has a pole at $E$ for some $\left.\psi \in S\right\}$ coincides with $\sigma(H(\beta, \theta))$.

The proof of this theorem is to be obtained through the following two lemmas. For the sake of simplicity let us take for $S$ the linear hull of the Hermite functions, which are dilation analytic for $|\operatorname{Im} \theta|<\pi / 2$.

Lemma 3.9. Let $0<\operatorname{Im} \theta<\min \left(\frac{\pi}{4}, \frac{2 \pi}{2 n+3}\right), \psi \in S$, and $F(t)$ be any spectral function of $\bar{H}(\beta)$. For $n=0,1, \ldots$ define

$$
\mu_{n}(\psi)=\left\langle\psi(\bar{\theta}),[H(\beta, \theta)]^{n} \psi(\theta)\right\rangle,
$$

where as usual $\psi(\theta)=U(\theta) \psi$. Then:

$$
\mu_{n}(\psi)=\left\langle\psi,[H(\beta)]^{n} \psi\right\rangle=\int_{-\infty}^{+\infty} t^{n} d\langle\psi, F(t) \psi\rangle
$$

and the sequence $\left\{\mu_{n}(\psi)\right\}_{0}^{\infty}$ gives rise to an indeterminate Hamburger moment problem, i.e. there are infinitely many distinct positive measures $\varrho$ on $\mathbb{R}$ such that

$$
\mu_{n}(\psi)=\int_{-\infty}^{+\infty} x^{n} d \varrho(x), \quad n=0,1, \ldots
$$

Proof. If $\psi \in S, \psi \in \bigcap_{n=0}^{\infty} D\left(H(\beta)^{n}\right)$, and $\psi(\theta) \in \bigcap_{n=0}^{\infty} \mathrm{D}\left(\mathrm{H}(\beta, \theta)^{n}\right)$. Hence the first equality in (3.8) is an immediate consequence of the scaling since $H(\beta, \theta)$ is represented by the differential expression $e^{-\theta}\left(p^{2}+e^{2 \theta} x_{+}^{2} \beta e^{1 / 2}\left({ }^{2 n+3}\right)^{\theta} x^{2 n+1}\right)$ on $S$, which is an invariant domain for $H(\beta, \theta), \operatorname{Im} \theta>0$. Furthermore the second equality follows by (3.4) again because $S$ is an invariant domain for $H(\beta)$. Finally, let $\varrho_{a}(t)=\left\langle\psi, E_{a}(t) \Psi\right\rangle$ where $E_{a}(t)$ is the spectral family of a self-adjoint extension $H_{a}(\beta)$ of $H(\beta)$. Then any measure $\varrho_{a}(t)$, as well as any convex combination of them, solves the moment problem (3.9). This proves the lemma.

Lemma 3.10. Let $\psi \in S$, and $L_{\psi}^{2}$ be the subspace of $L^{2}$ defined as the closure of the linear hull $M_{\psi}$ of all vectors $H(\beta)^{n} \psi, n=0,1, \ldots$ Let $H_{\psi}(\beta)$ be the closure of $H(\beta) \uparrow M_{\psi}$. Then $(a) H_{w}(\beta)$ is a closed symmetric operator with deficiency indices indices $(1,1)$. (b) There is a spectral function $F_{\psi}(t)$ of $H_{\psi}(\beta)$ such that

$$
\begin{aligned}
f_{\psi}(E) & =\left\langle\psi(\bar{\theta}),[H(\beta, \theta)-E]^{-1} \psi(\theta)\right\rangle \\
& =\left\langle\psi, \mathscr{R}_{\psi}(E) \psi\right\rangle \equiv \int_{-\infty}^{+\infty}(t-E)^{-1} d\left\langle\psi, F_{\psi}(t) \psi\right\rangle,
\end{aligned}
$$

where $\mathscr{R}_{\psi}(E)$ is the generalized resolvent of $H_{\psi}(\beta)$ generated by the spectral function $F_{\psi}(t)$.

Proof. By the well known connection between symmetric operators cyclically generated and the classical moment problem (see e.g. [1], Chap. 4) $H_{\psi}(\beta)$ cannot be self-adjoint, because in this case the moment problem (3.9) would be determinate, and hence its deficiency indices must be $(1,1)$. To see (b), let us first remark that, by standard dilation analyticity arguments (see e.g. [9] for an analogous situation) 
$f_{\psi}(E)$ does not depend on $\theta$, so that it has a limit as $\theta_{2}=\operatorname{Im} \theta \rightarrow 0$. Furthermore $f_{\psi}(E)$ admits the series $\sum_{n=0}^{\infty} \mu_{n}(\psi) E^{-n}$ as an asymtotic expansion near $E=\infty, \mu_{n}(\psi)$ being defined by (3.7) for any $n$. Since the Hamburger moment problem (3.9) has solutions, by a well known result (see e.g. [1], Chap. 3) there is a positive measure $\varrho(t)$ on $\mathbb{R}$, whose moments are given by (3.9), such that $f_{\psi}(E)=\int_{-\infty}^{+\infty}(t-E)^{-1} d \varrho(t)$. On the other hand, since $H_{\psi}(\beta)$ is cyclically generated, it is also well known ([1], Chap. 4) that all such measures are given by $\varrho(t)=\left\langle\psi, F_{\psi}(t) \psi\right\rangle$, where $F_{\psi}(t)$ ranges over the set of all spectral functions of $H_{\psi}(\beta)$. This concludes the proof of Lemma 3.10 .

Proof of Theorem 3.8. Let us first remark that, given any single eigenvalue $E(\beta)$ of $H(\beta, \theta)$, there is at least a $\psi \in S$ such that $f_{\psi}(E)$ has a pole at $E(\beta)$. This is because the corresponding eigenprojection is one dimensional, and $\{\psi(\bar{\theta})\}$ is of course dense in $L^{2}$ as well as $\{\psi(\theta)\}$. Then the theorem will be proved if we can show the existence of a unique spectral function $F(t)$ of $\bar{H}(\beta)$ such that

$$
f_{\psi}(E)=\int_{-\infty}^{+\infty}(t-E)^{-1} d\langle\psi, F(t) \psi\rangle .
$$

As a matter of fact given (3.11) the assertion is true because, by the known spectral properties of $H(\beta, \theta)$, the 1.h.s. is a meromorphic function of $E$ in the whole complex plane, while the r.h.s. is a priori analytic for $\operatorname{Im} E>0$. Now given $\psi$ and a spectral function $F_{\psi}(t)$ of $H_{\psi}(\beta)$ in $L_{\psi}^{2}$, one can always find a spectral function $F(t)$ of $\bar{H}(\beta)$ in $L^{2}$ such that $F(t) \uparrow L_{\psi}^{2}=F_{\psi}(t)$. Hence, by $(3.10)$ :

$$
f_{\psi}(E)=\int_{-\infty}^{+\infty}(t-E)^{-1} d\langle\psi, F(t) \psi\rangle .
$$

Letting $\psi$ range over $S$, by the linearity of $f_{\psi}(E)$ the spectral function $F(t)$ cannot depend on $\psi$. Hence (3.11) holds and the theorem is proved.

Corollary 3.11. Let $\beta \in \mathbb{R}_{+}$, and $E(\beta)$ be an eigenvalue of $H(\beta, \theta)$, $0<\operatorname{Im} \theta<\min \left(\frac{\pi}{4}, \frac{2 \pi}{2 n+3}\right)$. Then, except for a discrete set of points on $\mathbb{R}_{+}$, $\operatorname{Im} E(\beta)<0$.

Proof. By Theorem 3.3 we have $\operatorname{Im} E(\beta) \neq 0$ except for a discrete set of values of $\beta$. Hence $\operatorname{Im} E(\beta)<0$ whenever $\operatorname{Im} E(\beta) \neq 0$ by the above result.

Corollary 3.12. Let $\beta \in \mathbb{R}_{+}$, and $f_{\psi}(E)$ be such that its meromorphic continuation to $\operatorname{Im} E \leqq 0$ has at least a pole for $\operatorname{Im} E<0$. Then the analytic continuation of $f_{\psi}(E)$ along any path crossing the real axis is not single valued.

Proof. As is well known, formula (3.6) a priori defines two analytic functions of $E$, for $\operatorname{Im} E>0$ and for $\operatorname{Im} E<0$ respectively. If these functions are the analytic continuation of one another, this continuation has to be many valued since we know by Theorem 3.8 that there is an analytic continuation of $f_{\psi}(E), \operatorname{Im} E>0$, which has at least a pole for $\operatorname{Im} E<0$. This proves the corollary. 
Remark. The above Corollary represents the exact statement of the above mentioned notion of second sheet pole of a unique generalized resolvent.

Acknowledgements. It is a plesure to thank Professor V. Grecchi for several useful suggestions, and Professor A. Grossmann for this interest in this work. We are also grateful to Professors J.J. Loeffel and B. Simon for having pointed out two errors in an earlier version of this work.

\section{Appendix}

To prove Theorem 2.13 for all $n \geqq 1$, we need two preliminary propositions. Let $\delta>0, \operatorname{Re} \gamma>0$, and $H(\gamma, \delta,|\beta|), H(\gamma, \delta)$ be defined as operators in $L^{2}$ by

$$
\begin{aligned}
& D(H(\gamma, \delta))=D\left(p^{2}\right) \cap D\left(x^{2 n}\right), \\
& \mathrm{H}(\gamma, \delta) u=\left(p^{2}+\gamma x^{2}+\delta x^{2 n}\right) u, \\
& D(H(\gamma, \delta,|\beta|))=D\left(p^{2}\right) \cap D\left(x^{2 n+1}\right), \\
& H(\gamma, \delta,|\beta|) u=\left(p^{2}+\gamma x^{2}+\delta x^{2 n}+i|\beta| x^{2 n+1}\right) u,
\end{aligned}
$$

respectively. Then we have:

Lemma A.1. Let $\delta>0, \operatorname{Re} \gamma>0$. Then there is $a b>0$ such that

$$
\begin{aligned}
& \left\|\left(p^{2}+\gamma x^{2}+i|\beta| x^{2 n+1}\right) u\right\|^{2}+\delta^{2}\left\|x^{2 n} u\right\|^{2} \\
& \quad \leqq 2\left\|\left(p^{2}+\gamma x^{2}+\delta x^{2 n}+i|\beta| x^{2 n+1}\right) u\right\|^{2}+b\|u\|^{2}
\end{aligned}
$$

for any $u \in D\left(p^{2}\right) \cap D\left(x^{2 n+1}\right)$. The positive constant $b$ can be chosen independent of $|\beta|$, $\delta$ and $\gamma$ for $0 \leqq|\beta|<\beta_{0}, 0 \leqq \delta<\delta_{0}, \gamma \in \Omega$, respectively. Here $\beta_{0}$ and $\delta_{0}$ are fixed positive numbers, and $\Omega$ is an arbitrary compact of the half-plane Re $\gamma>0$.

Proof. As quadratic forms on $D\left(p^{2}\right) \cap D\left(x^{2 n+1}\right) \otimes D\left(p^{2}\right) \cap D\left(x^{2 n+1}\right)$ we can write

$$
\begin{aligned}
\left(p^{2}+\bar{\gamma}\right. & \left.x^{2}+\delta x^{2 n}-i|\beta| x^{2 n+1}\right)\left(p^{2}+\gamma x^{2}+\delta x^{2 n}+i|\beta| x^{2 n+1}\right) \\
= & \left(p^{2}+\bar{\gamma} x^{2}-i|\beta| x^{2 n+1}\right)\left(p^{2}+\gamma x^{2}+i|\beta| x^{2 n+1}\right)+\delta^{2} x^{4 n}+\delta x^{2 n}\left(p^{2}+\operatorname{Re} \gamma x^{2}\right) \\
& +\delta\left(p^{2}+\operatorname{Re} \gamma x^{2}\right) x^{2 n}=\left(p^{2}+\bar{\gamma} x^{2}-i|\beta| x^{2 n+1}\right)\left(p^{2}+\gamma x^{2}+i|\beta| x^{2 n+1}\right)+\delta^{2} x^{4 n} \\
& +2 \delta \operatorname{Re} \gamma x^{2 n+2}+\delta\left[p,\left[p, x^{2 n}\right]\right]+2 \delta p x^{2 n} p \\
\geqq & \left(p^{2}+\bar{\gamma} x^{2}-i|\beta| x^{2 n+1}\right)\left(p^{2}+\gamma x^{2}+i|\beta| x^{2 n+1}\right)+\delta^{2} x^{4 n}-2 n(2 n-1) \delta x^{2 n-2} \\
\geqq & \frac{1}{2}\left(p^{2}+\bar{\gamma} x^{2}-i|\beta| x^{2 n+1}\right)\left(p^{2}+\gamma x^{2}+i|\beta| x^{2 n+1}\right)+\frac{1}{2} \delta^{2} x^{4 n}-b+\left(\frac{1}{2} \delta^{2} x^{4 n}\right. \\
& \left.-2 n(2 n-1) \delta x^{2 n-2}+b\right) \geqq \frac{1}{2}\left(p^{2}+\bar{\gamma} x^{2}-i|\beta| x^{2 n+1}\right)\left(p^{2}+\gamma x^{2}+i|\beta| x^{2 n+1}\right) \\
& +\frac{1}{2} \delta^{2} x^{4 n}-b
\end{aligned}
$$

for a suitable $b>0$, which can be chosen independent of $|\beta|$ and $\gamma$ as it is seen at once. To show the independence also of $\delta$, it is enough to have $\frac{1}{2} \delta^{2} x^{4 n}-2 n(2 n-1) \delta x^{2 n-2}+b \geqq 0$ for all $\delta$ such that $0 \leqq \delta \leqq \delta_{0}$. This condition is a fortiori satisfied if there is a $b$ such that $\frac{1}{2} \delta^{2} x^{4 n}$ $-2 n(2 n-1) \delta x^{2 n}-2 n(2 n-1) \delta_{0}+b \geqq 0$ for all $\delta, 0 \leqq \delta \leqq \delta_{0}$, which is trivially verified. This proves the lemma. 
Theorem A.1. $H(\gamma, \delta,|\beta|)$ is an $m$-accretive operator in $L^{2}$. If $R_{\gamma}(\delta,|\beta|, E)$ denotes the resolvent of $H(\gamma, \delta,|\beta|)$, and $R_{\gamma}(\delta, E)$ the resolvent of $H(\gamma, \delta)$, we have, uniformly on compacts in $\operatorname{Re} \gamma>0$ :

$$
\begin{array}{llc}
\left\|R_{\gamma}(\delta,|\beta|, E)-R_{\gamma}(\delta, E)\right\| \rightarrow 0 \quad \text { as } & |\beta| \rightarrow 0, \\
\left\|R_{\gamma}(\delta,|\beta|, E)-R_{\gamma}(|\beta|, E)\right\| \rightarrow 0 \quad \text { as } & \delta \rightarrow 0 .
\end{array}
$$

(A.4) holds for any fixed $\delta>0$, and (A.5) holds uniformly with respect to $|\beta|$ for $0 \leqq|\beta|<1$.

Proof. The first assertion is a consequence of the quadratic estimate (A.3) exactly as in Theorem 2.4. In addition, $H(\gamma, \delta)$ has compact resolvent as proved by Simon [17]. To see (A.4) and (A.5), first remark that by the same argument of Lemma 2.12 there are values of $E$ such that $\left\|R_{\gamma}(\delta,|\beta|, E)\right\|$ is bounded for $0 \leqq \delta \leqq \delta_{0}$, $0 \leqq|\beta| \leqq \beta_{0}$, uniformly on compacts in $\gamma$. Hence, proceeding as Lemma II.10.1 of [17], to prove (A.4) we need only to control the boundedness for $0 \leqq|\beta| \leqq \beta_{0}$, uniform over $\gamma$, of

$$
\Delta_{1}=\left[R_{\gamma}(\delta, E) x\right]\left[x^{2 n} R_{\gamma}(\delta,|\beta|, E)\right]
$$

because by the second resolvent formula $R_{\gamma}(\delta, E)-R_{\gamma}(\delta,|\beta|, E)=-|\beta| \Delta_{1}$. Now the boundedness of $R_{\gamma}(\delta, E) x$, with the required uniformity, is a particular case of results of [17], Sect. III.1, and that of $x^{2 n} R_{\gamma}(\delta,|\beta|, E)$. with the required uniformities, is for any fixed $\delta>0$ an immediate consequence of the quadratic estimate (A.3). Hence (A.4) is proved. To show (A.5), remark that again by the second resolvent formula we have $R_{\gamma}(|\beta|, E)-R_{\gamma}(\delta,|\beta|, E)=-\Delta_{2}$, where

$$
\Delta_{2}=\left[R_{\gamma}(|\beta|, E) x^{2}\right]\left[x^{2 n-2} R_{\gamma}(\delta,|\beta|, E)\right] \delta .
$$

Now $R_{\gamma}(|\beta|, E) x^{2}$ is bounded for $0 \leqq|\beta| \leqq \beta_{0}$, uniformly with respect to $\gamma$, by the quadratic estimate (2.6). Furthermore, by (A.3) $x^{2 n} R_{\gamma}(\delta,|\beta|, E)$ is bounded, uniformly with respect to $\gamma$, for $0 \leqq \delta \leqq \delta_{0}, 0 \leqq|\beta| \leqq \beta_{0}$. Since $\delta^{(n-1) n} x^{2 n-2}<\delta x^{2 n}+1$, the same is true for $\delta^{(n-1) / n} x^{2 n-2} R_{\gamma}(\delta,|\beta|, E)$. Hence $\Delta_{2}=O\left(\delta^{1 / n}\right)$ and the Theorem is proved.

Proof of Theorem 2.13. We have:

$$
\begin{aligned}
R_{\gamma}(O, E)-R_{\gamma}(|\beta|, E)= & R_{\gamma}(O, E)-R_{\gamma}(\delta, E)+R_{\gamma}(\delta, E)-R_{\gamma}(\delta,|\beta|, E) \\
& +R_{\gamma}(\delta,|\beta|, E)-R_{\gamma}(|\beta|, E) .
\end{aligned}
$$

Given $\varepsilon>0$, by Theorem A.2 and Theorem III.1 of [17] there is $\delta>0$, independent of $|\beta|$, such that $\left\|R_{\gamma}(O, E)-R_{\gamma}(\delta, E)\right\|<\varepsilon / 3,\left\|R_{\gamma}(\delta,|\beta|, E)-R_{\gamma}(|\beta|, E)\right\|<\varepsilon / 3$. Since $\delta$ does not depend on $|\beta|$, always by Theorem A. 2 there are values of $|\beta|$ so small that $\left\|R_{\gamma}(\delta, E)-R_{\gamma}(\delta,|\beta|, E)\right\|<\varepsilon / 3$, again uniformly with respect to $\gamma$. Hence, given $\varepsilon>0$, there are values of $|\beta|$ so small that

$$
\begin{aligned}
\left\|R_{\gamma}(O, E)-R_{\gamma}(|\beta|, E)\right\| \leqq & \left\|R_{\gamma}(O, E)-R_{\gamma}(\delta, E)\right\|+\left\|R_{\gamma}(\delta, E)-R_{\gamma}(\delta,|\beta|, E)\right\| \\
& +\left\|R_{\gamma}(\delta,|\beta|, E)-R_{\gamma}(|\beta|, E)\right\|<\varepsilon / 3+\varepsilon / 3+\varepsilon / 3=\varepsilon,
\end{aligned}
$$

and the theorem is proved. 


\section{References}

1. Akhiezer, N.I.: The classical moment problem. Edinburgh: Oliver and Boyd 1965

2. Akhiezer, N.I., Glazman, I.M.: Theory of linear operators in Hilbert space, Vol. II. New York: Ungar 1963

3. Baslev, E., Combes, J.M.: Commun. Math. Phys. 22, 280 (1971)

4. Bender, C.M., Wu, T.T.: Phys. Rev. 184, 1231 (1969)

5. Davydov, A.: Quantum mechanics. Oxford, New York: Pergamon Press 1965

6. Gradshtein, I.S., Ryzhik, I.M.: Tables of series, integral, and products. New York: Academic Press 1964

7. Graffi, S., Grecchi, V.: Commun. Math. Phys. 62, 83 (1978)

8. Hardy, G.H. : Divergent series. Oxford, UK: Oxford University Press 1947

9. Herbst, I.W.: Commun. Math. Phys. 64, 179 (1979)

10. Howland, J.S. : Pac. J. Math. 55, 157 (1974)

11. Kato, T.: Perturbation theory for linear operators. Berlin, Heidelberg, New York: Springer 1966

12. Loeffel, J.J., Martin, A., Simon, B., Wightman, A.S. : Phys. Lett. 30B, 656 (1969)

13. Loeffel, J.J., Martin, A.: Proc. Programme 25th Conference, Strasbourg 1970

14. Naimark, M.A.: Linear differential operators, Part II. London: Harrap 1964

15. Reed, M., Simon, B.: Methods of modern mathematical physics, Vol. II. New York: Academic Press 1975

16. Reed, M., Simon, B.: Methods of modern mathematical physics, Vol. IV. New York: Academic Press 1978

17. Simon, B.: Ann. Phys. 58, 76 (1970)

18. Simon, B.: The definition of molecular resonance curves by the method of exterior complex scaling. Phys. Rev. Lett. (to appear)

Communicated by J. Ginibre

Received October 10, 1979; in revised form March 12, 1980 